\title{
Dynamic Growth Pattern of Biophysical Parameters of Rapeseed-Mustard Cultivars under Different Thermal Environments and Row Orientation
}

\author{
Jagjeewan Singh*, Som Pal Singh and P.K. Kingra \\ School of Climate Change and Agricultural Meteorology, Punjab Agricultural University, \\ Ludhiana, Punjab, India \\ *Corresponding author
}

\section{A B S T R A C T}

Although the growth rate of biophysical parameters can be strongly influenced by row orientation and temperature during different phenological stages in Rapeseed-Mustard cultivars but the dynamics of plant growth is not fully understood. Hence, a field experiment was conducted at the Research farm, School of Climate Change and Agricultural Meteorology, PAU, Ludhiana to characterize the dynamic growth pattern of biophysical parameters of three Brassica cultivars under different thermal environments

\section{Keywords}

Crop growth rate, Dry matter, Leaf area ratio, Leaf weight ratio, Plant height, Rapeseed-

Mustard, Relative growth rate, Specific leaf area

\section{Article Info}

Accepted: 07 April 2018 Available Online: 10 May 2018 and row orientation. The accumulation of dry matter partitioning and plant height was same in October 7 sown crop as compared to October 17 and October 27 sown crop. Early sown crop recorded significantly highest plant height $(84.9 \mathrm{~cm}, 146.4 \mathrm{~cm}$ and $167.1 \mathrm{~cm})$ during vegetative, flowering and pod filling stages respectively as compared to the late sown crop. Among the cultivars, PBR 357 accumulated highest dry matter and attained maximum plant height $(176.5 \mathrm{~cm})$ as compared to GSC $7(165.2 \mathrm{~cm})$ and PC $6(152.8 \mathrm{~cm})$ cultivars at maturity stage. The highest leaf area index was observed in early sown crop as leaf area index was reduced with delay in sowing. Crop growth rate was higher in October $7\left(54.2 \mathrm{gm}^{-2} \mathrm{~d}^{-1}\right)$ sown crop followed by October $17\left(50.6 \mathrm{gm}^{-2} \mathrm{~d}^{-1}\right)$ and October $27(44.3$ $\left.\mathrm{gm}^{-2} \mathrm{~d}^{-1}\right)$ during pod filling stage. Among the cultivars, the growth rate of leaf dry matter was observed highest in PBR 357 followed by GSC 7 and PC 6 at vegetative and pod filling stages. The Relative growth rate increased throughout life cycle of crop rather than CGR was decreased after 100 of sowing. The leaf area ratio and specific leaf area were highest in PC 6 as compared to PBR 357 and GSC 7 but the leaf weight ratio and specific leaf weight observed highest in PBR 357 followed by PC 6 and GSC 7. No effect of row orientation was observed in dry matter accumulation, plant height and crop growth rate. The study concluded that alteration in sowing time is highly effective for better growth of biophysical parameters of Rapeseed-mustard cultivars and thus, can be used as adaptation strategy to manage high temperature effects under changing climatic scenarios.

\section{Introduction}

Rapeseed-mustard (Brassica spp. L.) being an oilseed crop is the important component of economy after the cereal crops in India. Out of the total area and production of India, after the food grain crops, Brassica spp. contribute second maximum after Soybean among the oilseed crops and India being the second largest cultivator after China (Hedge, 2005). 
But in the recent survey, to meet the demands of oilseeds, India still is a net importer of vegetable oils and almost $40 \%$ of its annual edible oil needs are met by import.

The genetic potential of any crop is greatly influenced by weather parameters (temperature, rainfall) which play crucial role in determining the crop growth, development and yield (Iraddi, 2008). Any significant deviation of weather from the optimum value became detrimental for the crop productivity. Both the maximum and minimum temperature during early vegetative phase and reproductive phase plays a pivotal role in the crop growth rate, dry matter accumulation and its partitioning and ultimately the yield of Brassica cultivars. Thus it is important to understand the component unit of crop stand, accumulated biomass as well as their performance in different thermal environments. Being a Rabi oilseed crop, the production efficiency of Brassica cultivars is going to be decrease because these are liable to small change in temperature. Mall et al., (2004) reported that due to larger increase in temperature and higher uncertainties in rainfall, the crop production in winter season become comparatively more vulnerable.

Plant growth estimate is the fundamental method to assess the effect of temperature on crop growth rate of different phenological stages throughout the life cycle of the crop (Sharifi and Zadeh, 2012). The plant components (dry matter partitioning, crop growth rate and relative growth rate etc.) play important contribution in ultimate yield of the crop. The primary data of components of plant (dry matter partitioning, crop growth rate and relative growth rate etc.) needs to be investigated to probe the effects of environment factors within the whole plant and crop yield. The effect of temperature and row orientation on the phenological behavior and dry matter production of the crop can be assessed by investigating the crop growth rate from one phenological stage to another phenological stage. Low temperature during early stages and high temperature during near maturity stage reduce the partitioning due to slower growth and by hastening the life cycle of crop respectively.

Thus, there is a need for optimizing the sowing time and row orientation in order to provide congenial temperature which is favorable for growth and development of crop. The present experiment was, therefore, undertaken to find out the optimum sowing time and row orientation for the Brassica cultivars based on the performance of their growth dynamics, dry matter production and its partitioning.

\section{Materials and Methods}

The experiment was conducted during Rabi season in 2015-16 at the Research Farm, School of Climate Change and Agricultural Meteorology, Punjab Agricultural University, Ludhiana, located in central Punjab at $30^{\circ}-54$ $\mathrm{N}$ latitude and $75^{\circ}-48^{\prime}$ E longitude at an altitude of $247 \mathrm{~m}$ above the mean sea level. The area is characterized by sub-tropical semiarid type of climate, which experiences very hot summers during April to June and cold winters from December to January with average annual rainfall of $760 \mathrm{~mm}$. The experiment composed of split-split design with three dates of sowing, $\mathrm{D}_{1}\left(7^{\text {th }}\right.$ October $)$, $\mathrm{D}_{2}\left(17^{\text {th }}\right.$ October $)$ and $\mathrm{D}_{3}\left(27^{\text {th }}\right.$ October $)$, three varieties i.e. $\mathrm{V}_{1}$ (PBR 357), $\mathrm{V}_{2}(\mathrm{PC} 6)$ and $\mathrm{V}_{3}$ (GSC 7) and two row orientations $\mathrm{O}_{1}$ (NorthSouth) and $\mathrm{O}_{2}$ (East-west). Thinning was done manually to maintain the plant to plant distance of $15 \mathrm{~cm}$ and weeding was done as per need. Dry matter accumulation and crop growth rate were measured during different growth stages of crop from 40 to 120 days after sowing. Daily maximum and minimum temperature during the entire growing period 
of crop under all the dates of sowing was recorded from the agro-meteorological observatory was averaged during different phenophases of the crop. Crop biometric parameters e.g. Plant height, leaf area index, dry matter production and its partitioning to root, stem, leaves and pods was recorded at 20 days interval starting from 40 DAS. Growth indices at periodic intervals were worked out according to Hunt (1989) as follows:

Crop Growth Rate (CGR)

$\operatorname{CGR}\left(\mathrm{g} \mathrm{m}^{-2} \mathrm{~d}^{-1}\right)=\frac{\mathrm{W}_{2}-\mathrm{W}_{1}}{-\cdot-\cdot---}$

Where, $\mathrm{W}_{2}$ and $\mathrm{W}_{1}$ are the total dry matter of crop $\left(\mathrm{g} / \mathrm{m}^{2}\right)$ on days $\mathrm{T}_{2}$ and $\mathrm{T}_{1}$, respectively.

\section{Relative Growth Rate (RGR)}

$\operatorname{RGR}\left(\mathrm{g} \mathrm{m}^{-2} \mathrm{~d}^{-1}\right)=\frac{\ln \mathrm{W}_{2}-\ln \mathrm{W}_{1}}{\mathrm{~T}_{2}-\mathrm{T}_{1}}$

Where, $\mathrm{InW}_{1}$ and $\mathrm{InW}_{2}$ is natural $\log$ of total dry matter of crop $\left(\mathrm{g} / \mathrm{m}^{2}\right)$ on days $\mathrm{T}_{1}$ and $\mathrm{T}_{2}$, respectively.

\section{Leaf area ratio (LAR)}

$\operatorname{LAR}\left(\mathrm{m}^{2} \mathrm{~g}^{-1}\right)=\frac{\mathrm{LA}}{\mathrm{W}}$

Where,

$\mathrm{LA}=$ Leaf area $\left(\mathrm{m}^{2}\right)$

$\mathrm{W}=$ Total plant dry weight $(\mathrm{g})$

Specific Leaf Area (SLA)

$$
\operatorname{SLA}\left(\mathrm{m}^{2} \mathrm{~g}^{-1}\right)=\frac{\text { LA }}{\mathrm{LW}}
$$

Where,

$\mathrm{LA}=$ Leaf area $\left(\mathrm{m}^{2}\right)$

$\mathrm{LW}=$ Leaf weight $(\mathrm{g})$

Leaf Weight Ratio (LWR)

LWR = ----------

Where,

LW = Leaf weight $(\mathrm{g})$

$\mathrm{W}=$ Plant dry weight $(\mathrm{g})$

Specific Leaf Weight (SLW)

$\operatorname{SLW}\left(\mathrm{g} \mathrm{m}^{-2}\right)=\frac{\mathrm{LW}}{\mathrm{LA}}$

Where,

$\mathrm{LW}=$ Leaf weight $(\mathrm{g})$

$\mathrm{LA}=$ Leaf area $\left(\mathrm{m}^{2}\right)$

\section{Results and Discussion}

\section{Maximum and minimum temperature}

In order to investigate the interactive effects of temperature on growth rate and dry matter partitioning, maximum and minimum temperature during crop growth under three dates of sowing is shown in figure 1. This meteorological data revealed that in early growth stages both the maximum and minimum temperature were higher in first date but after that both the maximum and minimum temperature in first date started decreasing and in second and third date of sowing it went on increasing from 80 to 100 days after sowing to physiological maturity. The average maximum and minimum temperature was higher during early growth stages in the crop sown on 
October 7. However the temperature during the reproductive stages was reported to be higher in crop sown on October 27 which might have caused forced maturity of crop (Table 1).

\section{Plant height}

There was a linear increase in plant height with zenith at 120 days after sowing and thereafter there was decline in plant height due to fact that complete loss of flowers happens late because of their indeterminate in nature (Table 2). Among the dates of sowing, the highest plant height was observed in $7^{\text {th }}$ October $(170.9 \mathrm{~cm})$ which was statistically at par with second date of sowing $(166.8 \mathrm{~cm})$ except at 80 days after sowing. The lowest plant height was observed in late sown crop $(156.8 \mathrm{~cm})$ and the trends remained same till 120 days after sowing. Kumar et al., (2010) reported that lower temperature during vegetative phase caused lowered plant height due to poor growth in late sown conditions $\left(27^{\text {th }}\right.$ October). Kaur and Sidhu (2004) reported that with the delay in sowing date from October to December reduced the plant height significantly in Brassica carinata. Among the varieties PBR 357 produced higher plant height throughout the life cycle of crop but the cultivar GSC 7 was statistically at par with PC 6. Among the row orientation treatment, the crop sown in N-S direction was statistically at par with crop sown in E-W direction from sowing to 80 days after sowing. At 120 days after sowing, N-S sown crop observed statistically higher plant height than crop sown in E-W direction. After that, the plant height under both the row directions was almost similar with each other.

\section{Leaf area index}

A linear increase was observed in leaf area index with maxima at 100 days after sowing and thereafter there was decline in leaf area index due to leaf senescence in all the treatments (Table 2). Among the date of sowing, early sown crop ( $7^{\text {th }}$ October) having highest leaf area index which was statistically at par with second date of sowing $\left(17^{\text {th }}\right.$ October). The lowest leaf area index was observed in late sown crop ( $27^{\text {th }}$ October) and the trends remained same till 120 DAS. Kumar et al., (2010) reported that lower temperature during vegetative phase caused lowered LAI due to poor growth in late sown conditions $\left(27^{\text {th }}\right.$ October).

Kaur and Sidhu (2004) reported that with the delay in sowing date from October to December the LAI was significantly reduced in Brassica carinata. Among the varieties, PBR 357 produced higher leaf area index at 40 DAS which was at par with GSC 7, both these varieties recorded higher leaf area index as compared to PC 6. Similar trend was observed up to 100 DAS but thereafter there is no significant difference between leaf area index of all the three cultivars. Among the row orientation treatments, N-S sown crop recorded highest leaf area index as compared to E-W sown crop. Similar trend was observed up to 80 DAS after that there was no significant difference between these two treatments. Jha et al., (2015) concluded that the highest leaf area index was obtained in east-west direction as compared to north-south direction due to higher growth rate.

\section{Dry matter accumulation and its partitioning}

There was steady increase in dry matter (DM) with advancing crop growth. The maximum increase in dry matter was observed during 80 to 100 days after sowing (Fig. 2). Early sown ( $7^{\text {th }}$ October) crop accumulated highest root dry matter which was at par with mid-October $\left(17^{\text {th }}\right.$ October) sown crop and significantly higher than late sown crop $\left(27^{\text {th }}\right.$ October). Among the varieties and row direction 
treatments there was no significant difference between them.

A linear increase was observed in stem biomass with advanced phases of crop growth. At 40 days after sowing, the highest stem dry matter was accumulated by $7^{\text {th }}$ October (50.6 $\left.\mathrm{gm} / \mathrm{m}^{2}\right)$ followed by $17^{\text {th }}$ October (37.1 $\mathrm{gm} / \mathrm{m}^{2}$ ) and $27^{\text {th }}$ October $\left(31.0 \mathrm{gm} / \mathrm{m}^{2}\right)$ sown crop. Similar trend was observed up to 120 DAS. Among the varieties, the highest stem biomass was observed in GSC 7 followed by PBR 357 and PC 6 at 40 DAS. At 60 DAS, no significant difference was observed among varieties. However, from 80 to 120 DAS the highest stem biomass was obtained in GSC 7 followed by PC 6 and PBR 357. Between the rows orientation non-significant differences in stem dry matter within varieties were reported.

Among the dates of sowing, 40 DAS the highest leaves dry matter accumulation under early sown crop due to better growth followed by second and third date of sowing respectively. With delay in sowing there was reduction in leaf dry matter accumulation due to reduced plant growth.

Thereafter at 60 DAS, early and mid-sown crop leaves dry matter was higher than third $\left(27^{\text {th }}\right.$ October) date of sowing and similar trend was observed up to 120 DAS. At 40 DAS there were non-significant differences among the varieties. But at 60 DAS, PBR 357 recorded highest leaves dry matter followed by PC 6 and GSC 7. There after same trend was observed up to 120 DAS. With early sowing of crop the pod dry matter was obtained maximum and it decreased with delay in sowing. The highest pod dry matter was recorded in PBR 357 followed by GSC 7 and PC 6 from 80 DAS to 120 DAS.

The highest total dry matter accumulated in early sowing $\left(7^{\text {th }}\right.$ October) at 40 DAS and was followed by mid sown $\left(17^{\text {th }}\right.$ October) and late sown $\left(27^{\text {th }}\right.$ October). Thereafter at 60 DAS early and mid-sown crop were accumulate almost equal dry matter but higher than late sown crop. After that from 80 DAS to 120 DAS October 7 sown crop (2218.2 gm) was accumulated highest dry matter over October 17 (2066.5 gm) and October 27 (1733.1gm). Patel et al., (2013) also reported that the increased temperatures during late reproductive phase under delayed sowing hastened the maturity and therefore, the crop growth period was shortened resulting in reduced dry matter production as well as lesser partitioning into different plant parts. In comparison of varieties, at 40 DAS there was no significant difference in varieties. PBR 357 accumulated higher total dry matter which as compared to PC 6 and GSC 7 At 60 DAS and thereafter.

\section{Crop Growth Rate (CGR)}

The results showed that with delay in sowing the crop growth rate decreased significantly in root, leaves, stem and pod except leaf, stem and pod growth rate was non-significant at 4060 days after sowing, 80-100 days after sowing and 100-120 days after sowing respectively (Table 3). Among the three cultivar, root and stem dry matter growth rate were non-significant at all the stages except for the stem growth rate was significant higher in GSC 7 followed by PBR 357 and PC 6 at 60-80 DAS. The leaves growth rate was found significantly higher in PBR 357 followed by PC 6 and GSC 7 from 60-100 days after sowing but sowing after 40-60 days, there was no significant difference in leaf growth rate. At 100 DAS, the leaf growth rate was negative due to senescence of leaves. The total CGR was significantly higher in October 7 (54.2 g $\left.\mathrm{m}^{-2} \mathrm{~d}^{-1}\right)$ sown crop followed by October 17 $\left(50.6 \mathrm{~g} \mathrm{~m}^{-2} \mathrm{~d}^{-1}\right)$ and October $27\left(44.3 \mathrm{~g} \mathrm{~m}^{-2} \mathrm{~d}^{-1}\right)$ at 80 to 100 days after sowing. Among the cultivars, the total CGR was non-significantly higher in GSC 7 than PC 6 and PBR 357. 
Fig.1 Daily maximum and minimum temperature during life cycle under three dates of sowing
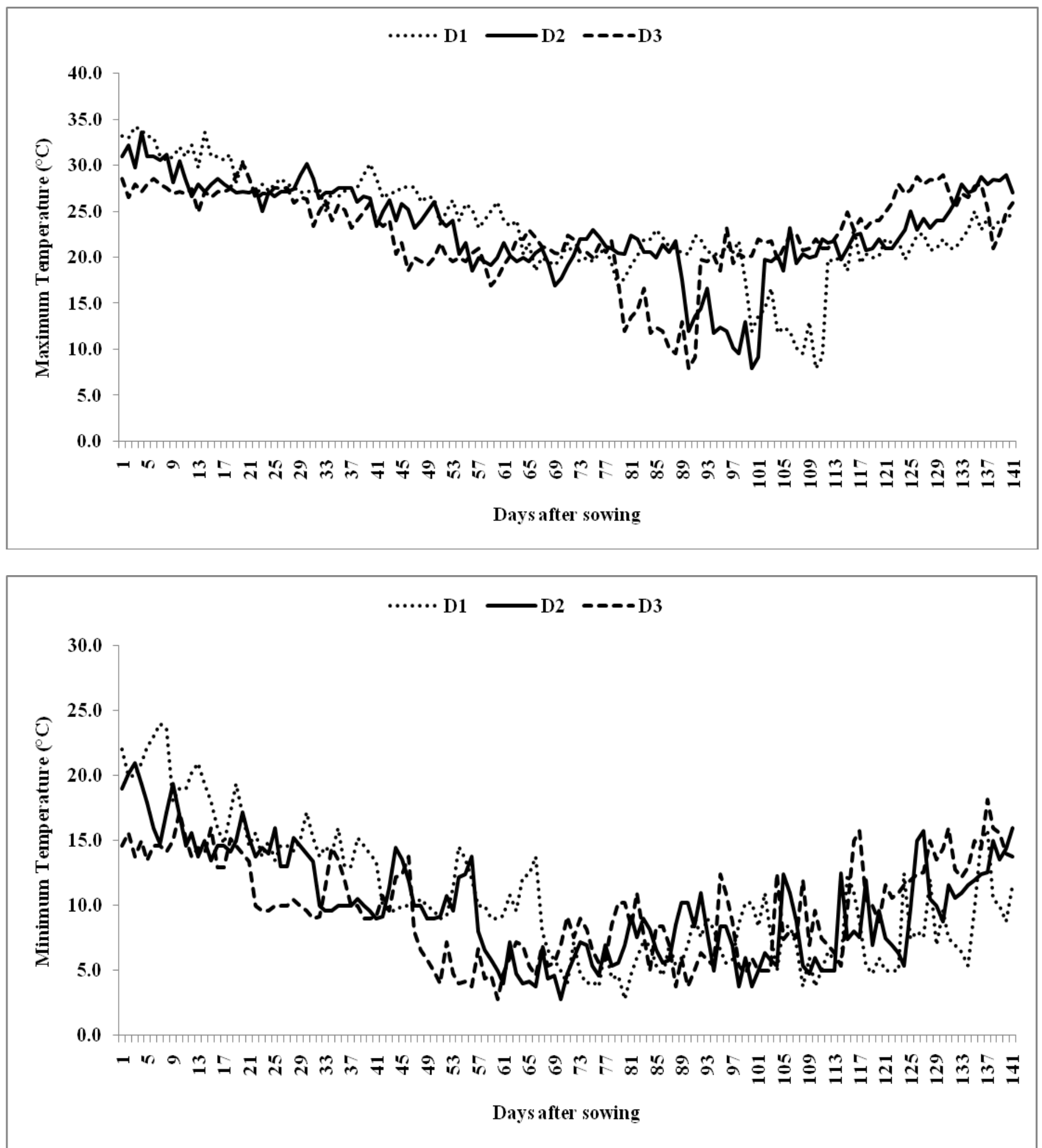
Fig.2 Dry matter accumulation under three thermal environments

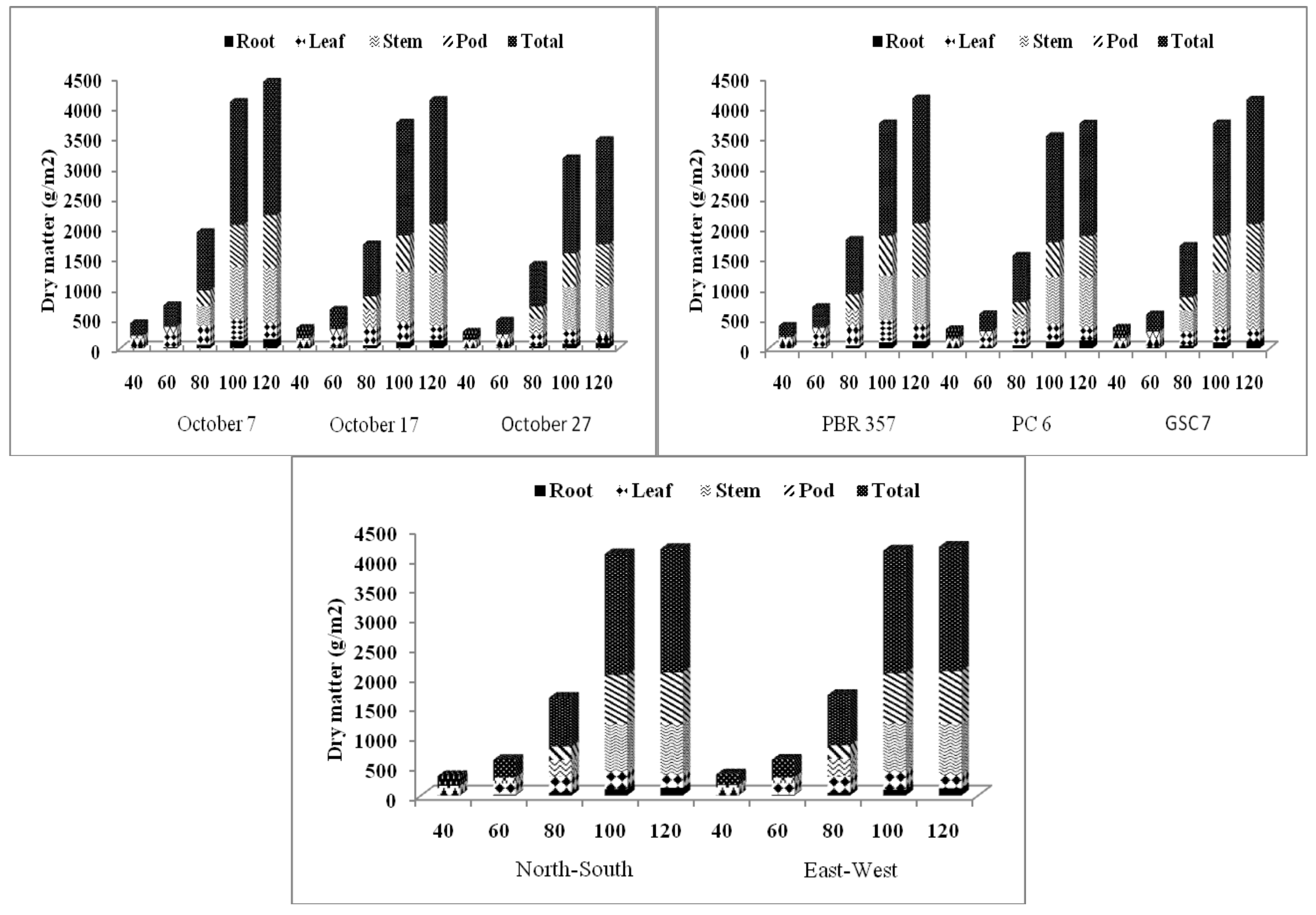


Table.1 Average temperature during phenological stages of crop under different thermal environments

\begin{tabular}{|c|c|c|c|c|c|c|}
\hline \multirow[t]{2}{*}{ Phenological stages } & \multicolumn{2}{|c|}{ October 7} & \multicolumn{2}{|c|}{ October 17} & \multicolumn{2}{|c|}{ October 27} \\
\hline & $\begin{array}{l}\text { Max. } \\
\left({ }^{\circ} \mathrm{C}\right)\end{array}$ & $\begin{array}{l}\text { Min } \\
\left({ }^{\circ} \mathrm{C}\right)\end{array}$ & $\begin{array}{l}\mathrm{Max} \\
\left({ }^{\circ} \mathrm{C}\right)\end{array}$ & $\begin{array}{l}\text { Min } \\
\left({ }^{\circ} \mathrm{C}\right)\end{array}$ & $\begin{array}{l}\mathrm{Max} \\
\left({ }^{\circ} \mathrm{C}\right)\end{array}$ & $\begin{array}{l}\text { Min } \\
\left({ }^{\circ} \mathrm{C}\right)\end{array}$ \\
\hline Sowing - Start emergence & 33.5 & 21.0 & 31.4 & 18.9 & 27.8 & 14.5 \\
\hline $\begin{array}{l}\text { Start emergence - Complete } \\
\text { emergence }\end{array}$ & 32.4 & 23.1 & 30.3 & 16.9 & 27.2 & 15.4 \\
\hline Complete emergence - 5 leaf stage & 31.2 & 21.2 & 28.4 & 16.1 & 26.8 & 14.8 \\
\hline 5 leaf stage - Start Flowering & 28.3 & 14.4 & 26.3 & 12.2 & 24.2 & 9.8 \\
\hline Start Flowering - Start Pod Formation & 22.6 & 9.4 & 20.0 & 6.4 & 20.7 & 6.1 \\
\hline Start Pod Formation -Start Pod Filling & 20.6 & 5.9 & 20.5 & 7.2 & 14.8 & 7.6 \\
\hline $\begin{array}{c}\text { Start Pod Filling Physiological } \\
\text { Maturity }\end{array}$ & 20.6 & 8.8 & 21.3 & 9.7 & 23.3 & 10.6 \\
\hline
\end{tabular}

Table.2 Plant height and leaf area index of Brassica varieties under different thermal environments and row orientations

\begin{tabular}{|c|c|c|c|c|c|c|c|c|c|c|c|}
\hline \multirow[t]{3}{*}{ Treatment } & \multicolumn{11}{|c|}{ Days after sowing } \\
\hline & 40 & 60 & 80 & 100 & 120 & Maturity & 40 & 60 & 80 & 100 & 120 \\
\hline & \multicolumn{6}{|c|}{ Plant height (cm) } & \multicolumn{5}{|c|}{ Leaf area index } \\
\hline \multicolumn{12}{|c|}{ Thermal environments } \\
\hline $7^{\text {th }}$ Oet & 42.6 & 84.9 & 146.4 & 167.1 & 191.1 & 170.9 & 1.03 & 1.62 & 1.96 & 2.36 & 2.01 \\
\hline $17^{\text {th }}$ Oct & 39.4 & 81.5 & 137.6 & 157.4 & 198.2 & 166.8 & 1.77 & 2.12 & 2.56 & 2.87 & 2.47 \\
\hline $27^{\text {th }}$ Oct & 21.7 & 67.1 & 113.8 & 147.9 & 158.7 & 156.8 & 1.72 & 2.00 & 2.43 & 2.82 & 2.39 \\
\hline $\begin{array}{c}\text { CD } \\
(p=0.05)\end{array}$ & 5.2 & 10.2 & 4.74 & 12.8 & 15.6 & 8.50 & 0.40 & 0.29 & 0.25 & 0.30 & 0.18 \\
\hline \multicolumn{12}{|c|}{ Varieties } \\
\hline PBR 357 & 43.02 & 117.7 & 165.8 & 171.2 & 190.8 & 176.5 & 1.68 & 2.05 & 2.46 & 2.84 & 2.38 \\
\hline PC 6 & 31.2 & 71.3 & 117.3 & 151.7 & 179.7 & 165.2 & 1.25 & 1.75 & 2.10 & 2.43 & 2.13 \\
\hline GSC 7 & 29.3 & 50.4 & 114.7 & 149.5 & 177.5 & 152.8 & 1.60 & 1.95 & 2.39 & 2.77 & 2.35 \\
\hline $\begin{array}{c}C D \\
(p=0.05) \\
\end{array}$ & 6.8 & 11.1 & 13.9 & 8.30 & 6.7 & 7.87 & 0.12 & 0.19 & 0.15 & 0.27 & $\mathrm{NS}$ \\
\hline \multicolumn{12}{|c|}{ Row orientation } \\
\hline N-S & 35.9 & 81.02 & 138.3 & 160.8 & 187.7 & 169.2 & 1.40 & 1.75 & 2.20 & 2.64 & 2.26 \\
\hline E-W & 33.1 & 74.6 & 126.9 & 154.2 & 177.7 & 160.4 & 1.61 & 2.08 & 2.44 & 2.72 & 2.32 \\
\hline $\begin{array}{c}C D \\
(p=0.05)\end{array}$ & NS & NS & NS & NS & 3.5 & 8.28 & 0.19 & 0.14 & 0.13 & NS & NS \\
\hline
\end{tabular}


Table.3 Crop growth rate of Brassica cultivars as affected by thermal environments and row orientation

\begin{tabular}{|c|c|c|c|c|c|c|c|c|c|c|c|c|c|c|c|c|c|c|}
\hline \multirow[t]{4}{*}{ Treatment } & \multicolumn{18}{|c|}{ Days after sowing } \\
\hline & \multicolumn{4}{|c|}{$40-60$} & \multicolumn{4}{|c|}{$60-80$} & \multicolumn{5}{|c|}{ 80-100 } & \multicolumn{5}{|c|}{$100-120$} \\
\hline & Root & Leaf & Stem & Total & Root & Leaf & Stem & Total & Root & Leaf & Stem & Pod & Total & Root & Leaf & Stem & Pod & Total \\
\hline & \multicolumn{18}{|c|}{ Thermal environments } \\
\hline $7^{\text {th }}$ Oct & 0.60 & 3.61 & 3.25 & 7.44 & 2.06 & 4.26 & 11.3 & 30.41 & 3.43 & 1.81 & 27.7 & 21.2 & 54.2 & 1.28 & - & 2.51 & 9.09 & 8.4 \\
\hline & & & & & & & & & & & & & & & 4.48 & & & \\
\hline $17^{\text {th }}$ Oct & 0.54 & 3.87 & 3.21 & 7.63 & 1.76 & 4.03 & 10.3 & 27.13 & 3.01 & 1.57 & 27.6 & 18.3 & 50.6 & 1.11 & $\begin{array}{c}- \\
4.14\end{array}$ & 2.09 & 10.4 & 9.4 \\
\hline $27^{\text {th }}$ Oct & 0.38 & 2.12 & 2.03 & 4.54 & 1.25 & 3.06 & 8.63 & 23.20 & 2.09 & 0.53 & 24.6 & 17.09 & 44.3 & 0.78 & 2.54 & 2.21 & 7.15 & 7.6 \\
\hline \multirow[t]{2}{*}{$\begin{array}{c}C D \\
(p=0.05)\end{array}$} & 0.14 & NS & 0.78 & NS & 0.40 & 0.86 & 1.46 & 1.83 & 0.67 & 0.84 & NS & 1.25 & 4.2 & 0.24 & 1.16 & 0.23 & NS & NS \\
\hline & \multicolumn{18}{|c|}{ Varieties } \\
\hline PBR 357 & 0.51 & 4.36 & 2.99 & 7.84 & 1.64 & 4.41 & 9.5 & 27.8 & 2.73 & 1.75 & 23.5 & 20.4 & 48.5 & 1.02 & $\begin{array}{c}- \\
4.47\end{array}$ & 2.20 & 11.6 & 10.3 \\
\hline PC 6 & 0.57 & 3.35 & 2.33 & 6.26 & 1.77 & 3.63 & 8.2 & 24.2 & 3.04 & 1.27 & 27.6 & 17.7 & 49.7 & 1.12 & $\begin{array}{c}- \\
3.58\end{array}$ & 2.20 & 5.51 & 5.25 \\
\hline GSC 7 & 0.44 & 1.89 & 3.17 & 5.51 & 1.65 & 3.32 & 12.4 & 28.5 & 2.76 & 0.89 & 28.7 & 18.5 & 50.9 & 1.03 & $\begin{array}{c}- \\
3.11\end{array}$ & 2.41 & 9.49 & 9.8 \\
\hline \multirow[t]{2}{*}{$\begin{array}{c}C D \\
(p=0.05) \\
\end{array}$} & NS & NS & NS & NS & NS & 0.73 & 2.64 & 3.48 & NS & 0.63 & NS & 1.35 & NS & NS & 0.98 & NS & NS & NS \\
\hline & \multicolumn{18}{|c|}{ Row orientation } \\
\hline $\mathbf{N}-\mathbf{S}$ & 0.54 & 3.41 & 2.91 & 6.85 & 1.77 & 3.76 & 9.50 & 26.3 & 2.95 & 1.30 & 26.8 & 18.8 & 49.9 & 1.10 & $\begin{array}{c}- \\
3.70\end{array}$ & 2.38 & 8.50 & 8.29 \\
\hline E-W & 0.47 & 2.99 & 2.75 & 6.22 & 1.61 & 3.80 & 10.6 & 27.4 & 2.74 & 1.31 & 26.5 & 18.9 & 49.4 & 1.01 & $\begin{array}{c}- \\
3.74\end{array}$ & 2.15 & 9.25 & 8.68 \\
\hline $\begin{array}{c}C D \\
(p=0.05)\end{array}$ & NS & NS & NS & NS & NS & NS & NS & NS & NS & NS & NS & NS & NS & NS & NS & 0.11 & $\mathrm{NS}$ & NS \\
\hline
\end{tabular}


Table.4 Relative growth rate of Brassica cultivars as affected by thermal environments and row orientation

\begin{tabular}{|c|c|c|c|c|c|c|c|c|c|c|c|c|c|c|c|c|c|c|}
\hline \multirow[t]{3}{*}{ Treatment } & \multicolumn{18}{|c|}{ Days after sowing } \\
\hline & \multicolumn{3}{|c|}{$40-60$} & \multirow[b]{2}{*}{ Total } & \multicolumn{3}{|c|}{$60-80$} & \multirow[b]{2}{*}{ Total } & \multicolumn{4}{|c|}{$80-100$} & \multirow[b]{2}{*}{ Total } & \multirow[b]{2}{*}{ Root } & \multicolumn{3}{|c|}{$100-120$} & \multirow[b]{2}{*}{ Total } \\
\hline & Root & Leaf & Stem & & Root & Leaf & Stem & & Root & Leaf & Stem & Pod & & & Leaf & Stem & Pod & \\
\hline \multicolumn{19}{|c|}{ Thermal environments } \\
\hline $7^{\text {th }}$ Oct & 2.80 & 5.11 & 4.49 & 5.59 & 3.92 & 5.42 & 5.56 & 6.56 & 4.62 & 5.51 & 6.50 & 6.24 & 7.27 & 4.77 & 5.20 & 6.50 & 6.42 & 8.40 \\
\hline $17^{\text {th }}$ Oct & 2.64 & 4.99 & 4.38 & 5.47 & 3.76 & 5.32 & 5.45 & 6.46 & 4.48 & 5.38 & 6.46 & 6.09 & 7.19 & 4.63 & 5.08 & 6.46 & 6.34 & 9.46 \\
\hline $27^{\text {th }}$ Oct & 2.19 & 4.68 & 4.05 & 5.15 & 3.31 & 5.02 & 5.26 & 6.26 & 4.02 & 5.03 & 6.29 & 6.03 & 7.03 & 4.16 & 4.77 & 6.30 & 6.18 & 7.60 \\
\hline $\begin{array}{c}\text { CD } \\
(p=0.05)\end{array}$ & 0.28 & NS & 0.21 & 0.22 & 0.28 & 0.30 & 0.15 & 0.10 & 0.30 & 0.35 & 0.13 & 0.06 & 0.06 & 0.29 & NS & 0.12 & 0.06 & NS \\
\hline \multicolumn{19}{|c|}{ Varieties } \\
\hline PBR 357 & 2.51 & 5.15 & 4.28 & 5.54 & 3.62 & 5.45 & 5.39 & 6.49 & 4.33 & 5.52 & 6.31 & 6.19 & 7.18 & 4.47 & 5.24 & 6.33 & 6.44 & 10.3 \\
\hline PC 6 & 2.60 & 4.89 & 4.15 & 5.32 & 3.71 & 5.21 & 5.26 & 6.34 & 4.43 & 5.26 & 6.39 & 6.06 & 7.13 & 4.58 & 4.98 & 6.39 & 6.18 & 5.25 \\
\hline GSC 7 & 2.53 & 4.74 & 4.48 & 5.35 & 3.65 & 5.10 & 5.62 & 6.45 & 4.36 & 5.14 & 6.54 & 6.10 & 7.19 & 4.51 & 4.84 & 6.54 & 6.32 & 9.8 \\
\hline $\begin{array}{c}\text { CD } \\
(p=0.05)\end{array}$ & NS & 0.30 & NS & NS & NS & 0.25 & 0.15 & 0.11 & NS & NS & 0.11 & 0.07 & 0.09 & NS & 0.28 & 0.09 & 0.13 & NS \\
\hline \multicolumn{19}{|c|}{ Row orientation } \\
\hline $\mathbf{N}-\mathbf{S}$ & 2.60 & 4.95 & 4.31 & 5.42 & 3.72 & 5.27 & 5.38 & 6.42 & 4.42 & 5.33 & 6.41 & 6.12 & 7.16 & 4.57 & 5.04 & 6.41 & 6.31 & 8.29 \\
\hline E-W & 2.49 & 4.91 & 4.30 & 5.39 & 3.61 & 5.24 & 5.47 & 6.44 & 4.33 & 5.29 & 6.43 & 6.13 & 7.17 & 4.47 & 5.01 & 6.43 & 6.33 & 8.68 \\
\hline $\begin{array}{c}C D \\
(p=0.05)\end{array}$ & NS & NS & NS & NS & NS & NS & NS & NS & NS & NS & NS & NS & NS & NS & NS & NS & NS & NS \\
\hline
\end{tabular}


Table.5 Leaf area ratio and specific leaf area of Brassica cultivars under different thermal environments and row orientation

\begin{tabular}{|c|c|c|c|c|c|c|c|c|c|c|}
\hline \multirow[t]{3}{*}{ Treatment } & \multicolumn{10}{|c|}{ Days after sowing } \\
\hline & 40 & 60 & 80 & 100 & 120 & 40 & 60 & 80 & 100 & 120 \\
\hline & \multicolumn{5}{|c|}{ Leaf area ratio } & \multicolumn{5}{|c|}{ Specific leaf area } \\
\hline \multicolumn{11}{|c|}{ Thermal environments } \\
\hline 7 Oct & 0.0095 & 0.0063 & 0.0027 & 0.0014 & 0.0011 & 0.013 & 0.010 & 0.009 & 0.009 & 0.010 \\
\hline 17 Oct & 0.0114 & 0.0071 & 0.0029 & 0.0015 & 0.0012 & 0.016 & 0.010 & 0.010 & 0.011 & 0.012 \\
\hline 27 Oct & 0.0082 & 0.0076 & 0.0029 & 0.0015 & 0.0012 & 0.012 & 0.012 & 0.010 & 0.013 & 0.014 \\
\hline $\mathrm{CD}(\mathrm{p}=0.05)$ & NS & NS & NS & NS & NS & NS & NS & NS & NS & NS \\
\hline \multicolumn{11}{|c|}{ Varieties } \\
\hline PBR 357 & 0.0074 & 0.0055 & 0.0024 & 0.0013 & 0.0010 & 0.010 & 0.008 & 0.007 & 0.007 & 0.008 \\
\hline PC 6 & 0.0114 & 0.0084 & 0.0033 & 0.0016 & 0.0013 & 0.016 & 0.013 & 0.011 & 0.012 & 0.013 \\
\hline GSC 7 & 0.0103 & 0.0071 & 0.0029 & 0.0015 & 0.0012 & 0.015 & 0.013 & 0.011 & 0.013 & 0.015 \\
\hline$C D(p=0.05)$ & 0.0027 & 0.0021 & 0.0004 & 0.0002 & 0.0002 & 0.004 & NS & 0.003 & NS & 0.005 \\
\hline \multicolumn{11}{|c|}{ Row orientation } \\
\hline $\mathbf{N}-\mathrm{S}$ & 0.0096 & 0.0062 & 0.0027 & 0.0015 & 0.0012 & 0.014 & 0.010 & 0.009 & 0.010 & 0.012 \\
\hline E-W & 0.0098 & 0.0078 & 0.003 & 0.0015 & 0.0011 & 0.014 & 0.013 & 0.010 & 0.012 & 0.012 \\
\hline$C D(p=0.05)$ & NS & NS & 0.0002 & NS & NS & NS & NS & NS & NS & NS \\
\hline
\end{tabular}

Table.6 Leaf weight ratio and specific leaf weight of Brassica cultivars under different thermal environments and row orientation

\begin{tabular}{|c|c|c|c|c|c|c|c|c|c|c|}
\hline \multirow[t]{2}{*}{ Treatment } & \multicolumn{10}{|c|}{ Days after sowing } \\
\hline & 40 & 60 & 80 & 100 & 120 & 40 & 60 & 80 & 100 & 120 \\
\hline & \multicolumn{5}{|c|}{ Leaf weight ratio } & \multicolumn{5}{|c|}{ Specific leaf weight } \\
\hline \multicolumn{11}{|c|}{ Thermal environments } \\
\hline 7 Oct & 0.70 & 0.61 & 0.31 & 0.16 & 0.11 & 99.3 & 116.9 & 127.6 & 125.6 & 103.0 \\
\hline 17 Oct & 0.73 & 0.62 & 0.32 & 0.16 & 0.11 & 78.3 & 107.6 & 119.0 & 116.3 & 101.5 \\
\hline 27 Oct & 0.72 & 0.62 & 0.29 & 0.13 & 0.09 & 103.3 & 91.6 & 106.3 & 94.4 & 86.4 \\
\hline $\mathrm{CD}(\mathrm{p}=0.05)$ & NS & NS & NS & NS & NS & NS & NS & NS & NS & NS \\
\hline \multicolumn{11}{|c|}{ Varieties } \\
\hline PBR 357 & 0.77 & 0.67 & 0.35 & 0.18 & 0.12 & 130.3 & 141.1 & 156.1 & 149.6 & 126.7 \\
\hline PC 6 & 0.71 & 0.64 & 0.32 & 0.15 & 0.11 & 77.05 & 93.8 & 104.2 & 101.7 & 89.6 \\
\hline GSC 7 & 0.67 & 0.54 & 0.25 & 0.12 & 0.08 & 73.6 & 81.2 & 92.5 & 85.0 & 74.5 \\
\hline $\mathrm{CD}(\mathrm{p}=0.05)$ & 0.05 & 0.07 & 0.05 & 0.04 & 0.02 & 37.4 & 36.2 & 32.5 & 36.5 & 28.4 \\
\hline \multicolumn{11}{|c|}{ Row orientation } \\
\hline $\mathbf{N}-\mathrm{S}$ & 0.71 & 0.62 & 0.31 & 0.16 & 0.11 & 98.01 & 115.2 & 123.3 & 113.7 & 94.9 \\
\hline E-W & 0.72 & 0.61 & 0.30 & 0.16 & 0.10 & 89.3 & 95.4 & 112.0 & 110.5 & 99.0 \\
\hline$C D(p=0.05)$ & NS & NS & NS & NS & NS & NS & NS & NS & NS & NS \\
\hline
\end{tabular}


Hokmalipour et al., (2011) also reported that the crop growth rate became negative after flowering stage due to senescence of leaves. Pod growth rate was significantly higher in early sown crop as compared to late sown because in early sown crop lower temperature coincides with pod filling stage rather than late sown crop. The higher temperature at reproductive phase shortened the life cycle of crop and hence total growth rate was reduced. Among the cultivars, the pod growth rate was observed significantly higher in PBR 357 and GSC 7 followed by PC 6. Between the row directions, there were non-significant differences in dry matter partitioning and crop growth rate.

\section{Relative Growth Rate (RGR)}

In relation to the initial dry weight of plant, the relative increase in plant dry matter dynamically is the Relative growth rate of plant.

The results were shown that the relative growth rate of root, stem and pods were increased towards maturity but in leaves it was decreases due to senescence of leaves near maturity as presented in Table 4 . With delay in sowing, the relative growth rate decreased in all the three cultivars. The maximum significant growth rate of 6.56 $\mathrm{g} \mathrm{m}^{-2} \mathrm{~d}^{-1}$ was observed in $7^{\text {th }}$ October sown crop as compared to 6.46 and $6.26 \mathrm{~g} \mathrm{~m}^{-2} \mathrm{~d}^{-1}$ in $17^{\text {th }}$ and $27^{\text {th }}$ October sown crop respectively at 60 to 80 DAS.

Among the cultivars, the maximum significant RGR was observed in GSC 7 (7.19 $\left.\mathrm{g} \mathrm{m}^{-2} \mathrm{~d}^{-1}\right)$ and least in PC $6\left(7.13 \mathrm{~g} \mathrm{~m}^{-2} \mathrm{~d}^{-1}\right)$ at 80 to 100 days after sowing. The RGR of leaves were found to decrease after 100 days of sowing except for the other components of plant. There were non-significant differences in relative growth rate between two row orientations.

\section{Leaf Area Ratio (LAR)}

The data on leaf area ratio (LAR), the ratio of leaf area to total plant weight for different growth periods of crop at 20 days interval is presented in Table 5. The LAR was maximum at 40 days after sowing in all the three cultivars under three dates of sowing. Among the dates, there were non-significant differences observed but the highest LAR was observed in PC $6\left(0.011 \mathrm{~m}^{2} \mathrm{~g}^{-1}\right)$ followed by GSC $7\left(0.010 \mathrm{~m}^{2} \mathrm{~g}^{-1}\right)$ and PBR 357 (0.0074 $\left.\mathrm{m}^{2} \mathrm{~g}^{-1}\right)$ throughout the life cycle of crop. The leaf area ratio was decreased after vegetative phase up to maturity.

\section{Specific Leaf Area (SLA)}

Specific leaf area (SLA) is the ratio of leaf area to leaf weight at different growth stages of crop at is presented in Table 5. The SLA was maximum at 40 days after sowing in all the three cultivars under three dates of sowing but decreased as the crop advanced towards maturity with increase in leaf weight. Among the dates, there were non-significant differences observed but the highest LAR was observed in cultivar PC $6\left(0.016 \mathrm{~m}^{2} \mathrm{~g}^{-1}\right)$ and was followed by GSC $7\left(0.015 \mathrm{~m}^{2} \mathrm{~g}^{-1}\right)$ and PBR $357\left(0.0010 \mathrm{~m}^{2} \mathrm{~g}^{-1}\right)$ throughout the life cycle of crop. The leaf area ratio was decreased after vegetative phase up to maturity.

\section{Leaf Weight Ratio (LWR)}

The data on leaf weight ratio (LWR), the ratio of leaf weight to total plant weight, from 40 DAS to maturity at 20 days interval are presented in Table 6 . The perusal of the data revealed that among the three cultivars, PBR 357 attained maximum leaf weight ratio $\left(0.77 \mathrm{~g} \mathrm{~m}^{-2}\right.$ day $\left.^{-1}\right)$ at 40 DAS followed by PC 6 $\left(0.71 \mathrm{~g} \mathrm{~m}^{-2}\right.$ day $\left.^{-1}\right)$ and GSC $7\left(0.67 \mathrm{~g} \mathrm{~m}^{-2}\right.$ day $\left.^{-1}\right)$ in all the three dates of sowing. From vegetative stage to towards physiological 
maturity the LWR found to decrease because most of the leaves were fallen due to senescence and partitioning of dry matter moved towards pods.

\section{Specific Leaf Weight (SLW)}

The data on specific leaf weight (SLW), the ratio of leaf weight to leaf area, from 40 DAS to maturity at 20 days interval are presented in Table 6. The perusal of the data revealed that the SLW was less during initial crop growth and it increased with the age of crop. This may be due to the fact that as the crop growth occurred, leaf thickness increased resulting in increase in SLW. Between the three cultivars, PBR 357 attained maximum specific leaf weight $\left(156.1 \mathrm{~g} \mathrm{~m}^{-2}\right.$ day $\left.^{-1}\right)$ at 80 DAS followed by PC $6\left(104.2 \mathrm{~g} \mathrm{~m}^{-2}\right.$ day $\left.^{-1}\right)$ and GSC $7\left(92.5 \mathrm{~g} \mathrm{~m}^{-2}\right.$ day $\left.^{-1}\right)$ in all the three dates. The reduction in maximum specific leaf weight was observed with delayed in sowing but among the three dates and row direction the differences were non-significant.

The present study concluded that sowing of Rapeseed-mustard cultivars on October 7 exhibited significantly higher plant dry matter, plant height and crop growth rate due to optimal thermal requirements for various phenological stages of crop. Dry matter accumulation and plant height of Brassica cultivars decreased with delay in sowing beyond October 7 .

The crop growth indices were also decreased with delay in sowing from October 7 to October 27. Among the cultivars, PBR 357 had higher crop growth rate but GSC 7 and PC 6 exhibited similar rate. The direction of sowing of varieties did not show any significant differences between them. Hence, optimum temperature will coincides with different phenological stage with changing date of sowing to accumulate more dry matter and higher crop growth rate.

\section{References}

Hedge, D. M., 2005. Oilseed scenario in India - past, present and future with special reference to rapeseed-mustard. In: Winter School on Advances in Rapeseed- Mustard Research Technology for Sustainable Production of Oilseeds, National Centre on Rapeseed-Mustard, Sewar, Bharatpur. pp. 1-15.

Hokmalipour, S., Tobe, A., Jafarzadeh, B. and Darbandi, M. H. 2011. Study of sowing date on some morphological traits of spring canola (Brassica napus L.) cultivars. World Applied Sci J 14: 53138.

Hunt, R. 1989. Basic Growth Analysis: Plant Growth Analysis forbeginners. Unwin Lyman $(\mathrm{Pb})$, London.

Iraddi, V. S. 2008. Response of mustard (Brassica juncea L. Czernj and Cosson) Varieties to date of sowing and row spacing in Northern transition zone of Karnataka. Thesis Submitted to the University of Agricultural Sciences, Dharwad In partial fulfilment of the requirements for the Degree of Master of science (Agriculture) In Agronomy. pp. 55-81.

Kaur, P., and Sidhu, M. S. 2004. Effect of sowing date, nitrogen level and row spacing on the growth and yield of African sarson (Brassica carinata A. Br.). J Res Punjab Agric Univ 41: 2734.

Kumar G, Chakravarty N. V. K., Adak, T., Madhu, M., Sena, D. R., Vishwakarma, A. K., Haldar, D., and Anuranjan. 2010. Radiation interception parameters in mustard and its modification due to Debranching. Ind J Soil Conserv 38: 205-11

Mall, R. K., Lal, M., Bhatia, V. S., Rathore, L.S., and Singh, R. 2004. Mitigating climate change impact on soybean 
productivity in India: a simulation study. Agric. Forest Meteorol. 121, 113-125.

Patel, N., Tyagi, P. K., and Shukla, K. C. 2013. Influence of sowing date and varieties on total biomass and its partitioning into different plant parts of
Indian mustard (Brassica juncea L.). $J$ Multidisciplinary Advance Res 2: 12-16. Sharifi, R. S., and Zadeh, N. N. 2012, Effects of plant density and row spacing on biomass production and some of physiological indices of corn (Zea maize L.) in second cropping. J Food Agric and Environ vol 10: 795-801

\section{How to cite this article:}

Jagjeewan Singh, Som Pal Singh and Kingra, P.K. 2018. Dynamic Growth Pattern of Biophysical Parameters of Rapeseed-Mustard Cultivars under Different Thermal Environments and Row Orientation. Int.J.Curr.Microbiol.App.Sci. 7(05): 435-448.

doi: https://doi.org/10.20546/ijcmas.2018.705.056 